\title{
Appareil
}

\section{Traitements audionumériques pour la guitare électrique amplifiée}

\section{Benoît Courribet}

\section{(2) OpenEdition}

1 Journals

\section{Édition électronique}

URL : http://journals.openedition.org/appareil/1036

DOI : 10.4000/appareil.1036

ISSN : 2101-0714

Éditeur

MSH Paris Nord

\section{Référence électronique}

Benoît Courribet, «Traitements audionumériques pour la guitare électrique amplifiée », Appareil [En ligne], 5 | 2010, mis en ligne le 10 juin 2010, consulté le 30 juillet 2020. URL : http:// journals.openedition.org/appareil/1036 ; DOI : https://doi.org/10.4000/appareil.1036

Ce document a été généré automatiquement le 30 juillet 2020.

\section{(c) (i) (9)}

Appareil est mis à disposition selon les termes de la Licence Creative Commons Attribution - Pas d'Utilisation Commerciale - Pas de Modification 4.0 International. 


\title{
Traitements audionumériques pour la guitare électrique amplifiée
}

\author{
Benoît Courribet
}

\section{Introduction}

1 Nous nous intéressons, dans cet article, à la possibilité d'utiliser l'ordinateur comme outil de traitement sonore pour la guitare électrique.

2 Afin d'intégrer l'outil informatique comme nouvel élément d'un "réseau " déjà constitué, nous portons notre attention plus particulièrement aux éléments de ce réseau qui interviennent dans ce qu'est, ou plutôt, ce que sont les sonorités de la guitare électrique ${ }^{1}$.

3 Bien évidemment si l'on considère le son de la guitare électrique de manière causale, le jeu de l'instrumentiste et les caractéristiques (lutherie, électronique, cordes...) de l'instrument tiennent une grande part dans la signature sonore de l'instrument. Cela est commun à tous les instruments acoustiques. Cependant, dans le cas des instruments amplifiés en général et de la guitare électrique en particulier, le prolongement de l'instrument par le système d'amplification est un élément majeur à considérer. Aussi, après avoir décrit le système d'amplification de la guitare électrique, nous étudierons la place des effets dans la chaîne d'amplification. Ensuite, nous proposerons d'intégrer l'ordinateur à ce système, et après avoir décrit les principales précautions à observer, nous présenterons quelques réalisations logicielles effectuées dans l'environnement Max/MSP2.

4 Cet article est accompagné d'exemples sonores et logiciels compilés dans une archive téléchargeable à l'adresse suivante: http://cicm.mshparisnord.org/courribet/ guitare_electrique.zip 


\section{Le système d'amplification de la guitare électrique}

$5 \quad$ La vibration des cordes de l'instrument est captée par les microphones (des capteurs électromagnétiques) et crée un signal électrique induit qui va être transmis au système d'amplification. Si dans la configuration la plus simple, ce système d'amplification est constitué d'un élément unique (combo amplifier), il convient de détailler les différents modules qui le composent et qui tiennent chacun une grande part dans la sonorité perçue de l'instrument.

6 Tout d'abord, le signal de l'instrument passe par un étage de préamplification, qui sert à adapter le signal électrique (en l'amplifiant) à la suite du système d'amplification. C'est dans cette partie du système d'amplification que l'on trouve potentiellement le plus de réglages pour « sculpter » le son de la guitare électrique ${ }^{3}$.

7 Ensuite, le signal est transmis, en sortie du préamplificateur, à la section d'amplification de puissance. Si le but de ce module est d'amplifier le signal pour lui permettre d'être envoyé à un transducteur électroacoustique et de ne pas intervenir sur le timbre de l'instrument, les technologies utilisées (amplification de puissance à tubes, par exemple) peuvent induire un comportement fortement non-linéaire et une modification du timbre de la guitare électrique.

8 Enfin, le signal dit de puissance est appliqué à un ou plusieurs haut-parleurs dont la fonction est de rendre audible le signal de la guitare électrique.

Figure 1 : schéma du système d'amplification de la guitare électrique

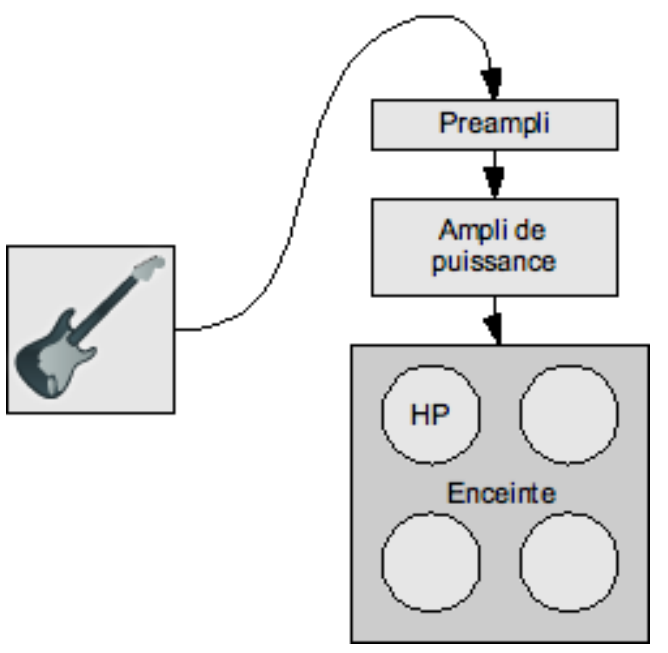

Dans le cas d'un amplificateur de type combo, tous ces éléments sont rassemblés dans un châssis unique. On trouve également des systèmes constitués d'un châssis réunissant le préamplificateur et l'amplificateur de puissance (dit «tête d'ampli») et d'une ou plusieurs enceintes, composées d'un ou plusieurs haut-parleurs placés dans un baffle. 
Figure 2a : photographie de combo

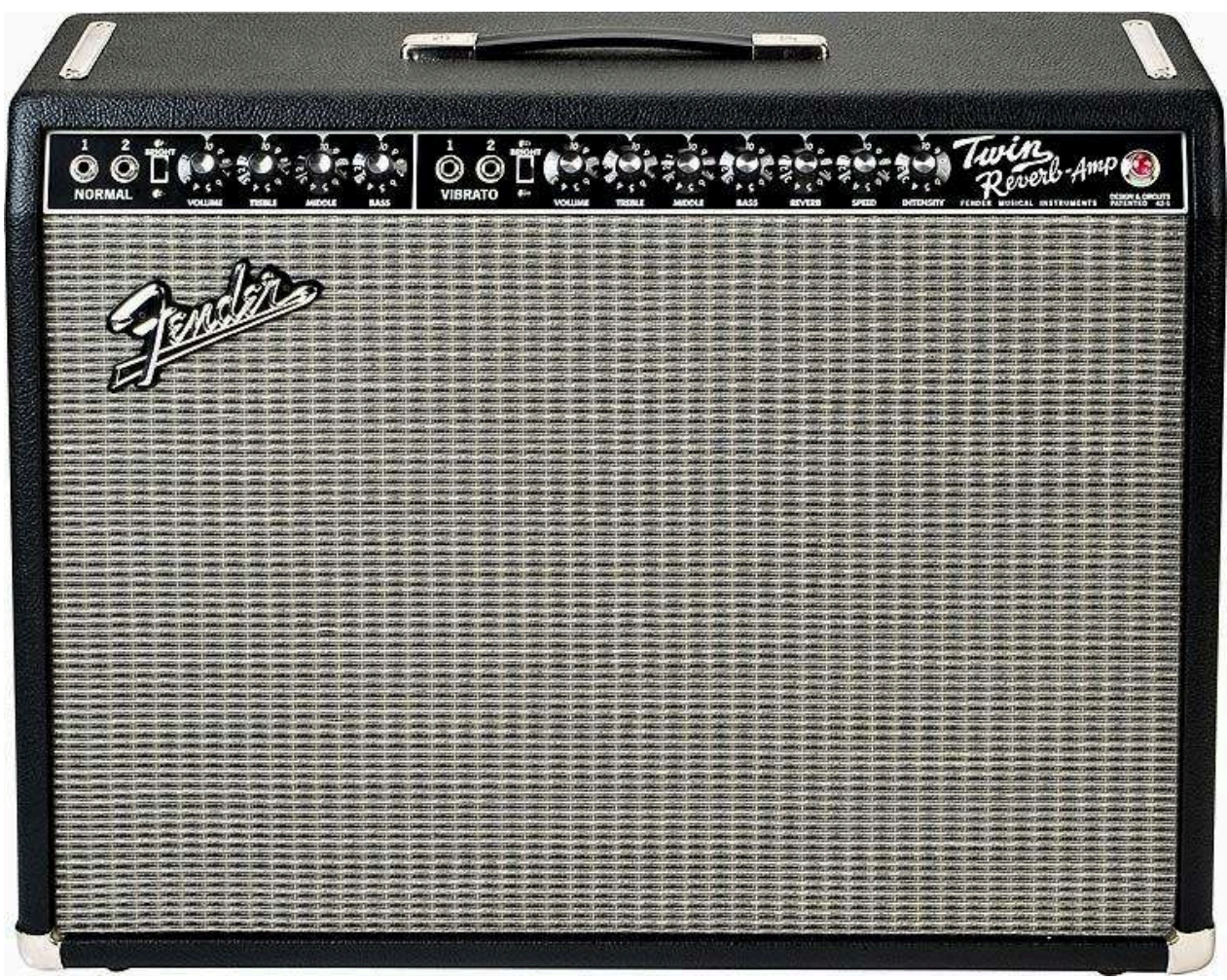

Figure $\mathbf{2 b}$ : photographie de tête d'ampli

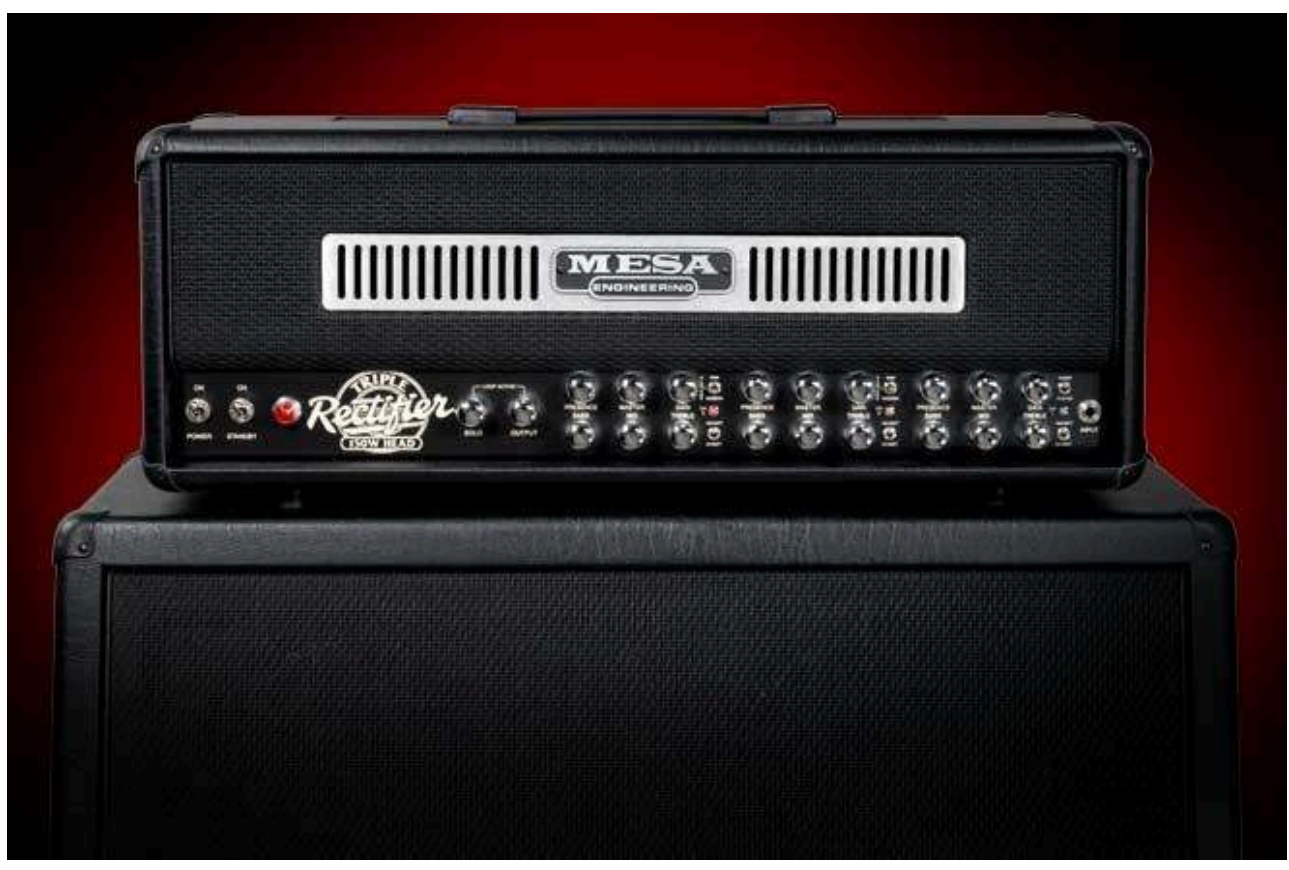

10 De la même manière que les différents éléments de la lutherie de la guitare électrique sont importants, les choix technologiques et spécificités de chacun des 3 éléments du système d'amplification auront leur importance dans la nature du son perçu ${ }^{4}$.

11 Il existe également d'autres paramètres qui vont venir influer sur le son de l'instrument. Tout d'abord, les conditions de diffusion du son en sortie de l'enceinte : 
les caractéristiques acoustiques de la pièce dans laquelle se trouve l'amplificateur, la disposition de l'amplificateur dans cette pièce (au sol, dans un coin, surélevé ou non...) et enfin la position de l'auditeur par rapport à l'ampli (en face ou de dos, dans l'axe des haut-parleurs ou non, à quelle distance...).

Ces dernières remarques valent pour la situation dans laquelle l'auditeur est présent dans le même espace acoustique que l'amplificateur ${ }^{5}$, ce qui s'avère une situation marginale, par rapport aux situations correspondant à l'écoute d'une œuvre musicale fixée sur support et diffusée.

Dans ce dernier cas, la méthode utilisée pour l'enregistrement ainsi que les différents paramètres associés, viendront apposer une "signature sonore" plus ou moins importante dans la sonorité finale de l'instrument. Parmi ces paramètres, on peut citer : choix du ou des microphones, positionnement du ou des microphones, choix du préampli micro, etc.

14 Enfin, lors du mixage d'un enregistrement contenant de la guitare électrique, le son enregistré est généralement l'objet de traitements comme par exemple l'égalisation et la compression dynamique.

15 Ainsi, nous voyons que le signal électrique issu de la guitare électrique traverse de nombreux étages de modification sonore avant d'être donné à entendre.

\section{La place des effets dans la chaîne d'amplification}

16 Les effets constituent un élément important du dispositif technologique associé à la guitare électrique. Dans la majorité des cas, qu'il s'agisse de dispositifs matériels (analogiques ou numériques) ou logiciels, les effets peuvent être considérés comme des filtres comportant une entrée et une sortie ${ }^{6}$.

Les effets doivent donc se placer sur le trajet du signal audio. Il existe plusieurs points permettant cette insertion :

- entre la guitare et l'entrée du préamplificateur

18 Ici se placent en général les pédales d'effet, activables et désactivables par l'intermédiaire d'un commutateur actionnable au pied. S'il n'existe pas de règle immuable quant au type de traitement que l'on peut insérer à cet endroit, on y place généralement les traitements de saturation (overdrive, distortion, fuzz), de compression, de génération d'octave et de synthèse.

- entre le préamplificateur et la section d'amplification de puissance

On trouve ici souvent les traitements de modulation (chorus, flanger, phaser, tremolo) et d'écho/réverbération. Pour les amplificateurs dont les sections de préamplification et de puissance sont réunies dans un même châssis, un point d'accès appelé «boucle d'effet » est parfois disponible. Il permet d'intercaler des dispositifs (pédales d'effet ou racks, multi-effets...) entre le préamplificateur et la section de puissance. La quantité de signal injectée dans cette boucle (ou balance entre son direct et son traité) est la plupart du temps réglable à l'aide d'un potentiomètre. 
Figure 3 : photographie des connecteurs et contrôle d'une boucle d'effets

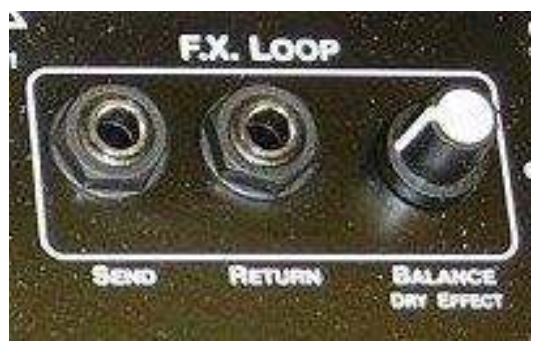

- après le préamplificateur de microphone captant le son de l'ampli guitare

On trouve ici les traitements qui sont rajoutés par l'ingénieur du son en studio lors du mixage, ou à la console de diffusion dans le cas d'un concert. Tous types de dispositifs et d'effets sont utilisables.

\section{Insertion de l'ordinateur comme dispositif de traitement audio dans la chaîne d'amplification}

21 Nous détaillons ici les principaux cas de figure permettant d'utiliser l'ordinateur comme processeur de traitements pour la guitare électrique.

\section{1. Le signal de la guitare est dévié de la chaîne d'amplification}

Par l'intermédiaire d'un dédoubleur de signal (splitter), le signal électrique émis par la guitare est dirigé d'une part vers le dispositif d'amplification de la guitare et d'autre part dans l'interface audionumérique reliée à l'ordinateur? ${ }^{7}$. Le signal traité par l'ordinateur sera par la suite diffusé sur un dispositif de diffusion indépendant de l'ampli guitare qui diffusera, quant à lui, le signal non traité.

23 Afin d'assurer au mieux l'intégrité du signal, il est préférable d'utiliser un boîtier de direct (ou DI pour Direct Injection) entre la guitare et l'interface audionumérique. Ce boîtier permet d'une part d'adapter l'impédance du signal à l'entrée de l'interface audionumérique et d'autre part, de symétriser le signal et de permettre ainsi une transmission optimale même en utilisant de grandes longueurs de câble ${ }^{8}$.

Enfin (et cela sera valable pour tous les cas de figures que nous allons détailler), il est important de noter que la configuration de l'interface audionumérique devra se faire avec comme objet de réduire au maximum la latence induite par le dispositif ${ }^{9}$. En fonction $\mathrm{du}$ traitement utilisé, une latence très faible (imperceptible par l'instrumentiste et/ou l'auditeur) ne sera pas forcément nécessaire.

\section{2. Le signal de la guitare est réinjecté dans la chaîne d'amplification après traitement}

Ici, l'ordinateur, par l'intermédiaire des entrées et sorties de l'interface audionumérique, va pouvoir être inséré sur le trajet du signal de la guitare.

- Entre la guitare et le préamplificateur :

La remarque précédente quant à l'utilisation d'un boîtier de direct reste valable et il faut également prendre soin d'opérer une réadaptation du signal sortant de l'interface 
audionumérique avant de le transmettre au préamplificateur. Un dispositif inverse du boîtier de direct, appelé boîtier de ré-amplification (le procédé est appelé re-amping) permet d'adapter le signal sortant de l'interface audionumérique au niveau ligne en le ramenant au niveau « instrument ».

- Entre les sections de préamplification et de puissance :

Il n'y a pas ici de précaution particulière à prendre quant à l'utilisation d'un ordinateur $^{10}$, le type de signal transmis entre le préamplificateur et la section de puissance peut-être transmis et récupéré de l'interface audionumérique sans adaptation.

- Après le préamplificateur du microphone captant le son de l'ampli guitare :

Ici encore, aucune précaution particulière n'est nécessaire, l'utilisation de traitements audionumériques sous la forme de plugins est chose courante dans le domaine de l'enregistrement en studio, le plugin s'insère dans un flux de données audio déjà numérisé.

\section{Exemples de traitements audionumériques pour la guitare électrique}

Nous allons maintenant présenter quelques réalisations logicielles permettant d'illustrer les différentes possibilités qu'offre l'utilisation de l'ordinateur comme dispositif de traitement audionumérique. Ces différents traitements s'inscrivent chacun dans une des trois catégories générales suivantes :

- la reproduction d'effets dits « classiques»;

- l'utilisation d'une analyse du signal entrant permettant un contrôle de plus haut niveau des paramètres de traitement ;

- l'extension multiphonique d'effets classiques, pour une diffusion sur dispositif multicanal.

On notera que nous nous sommes basés sur des traitements " classiques ", c'est-à-dire intégrés au catalogue de sonorités de la guitare électrique. Il est bien entendu possible d'utiliser d'autres types de traitements, notamment moins respectueux du timbre de la guitare électrique, mais ce n'est pas notre propos ici. 
Figure 4 : copie d'écran de l'interface graphique du Tremolo
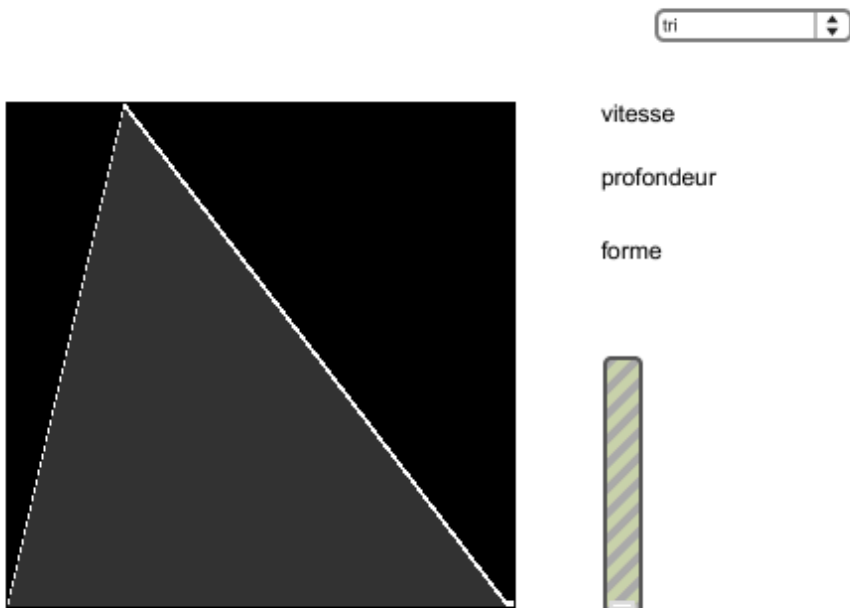

vitesse

profondeur

forme

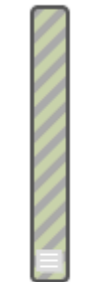

vers ampli

Х audio on/off

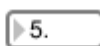

0.417

0.235

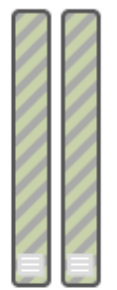

vers sono

\section{*Delay/Vibrato/Chorus/Flanger}

Plusieurs traitements peuvent être réalisés sur la base d'une ligne à retard.

Le premier est le Delay, effet permettant de mélanger un signal entrant à une version retardée dans le temps de ce même signal. La réinjection du signal dans la ligne à retard permet d'obtenir des échos multiples, décroissant en intensité dans le temps.

Figure 5 : implémentation d'une ligne à retard avec réinjection dans Max/MSP

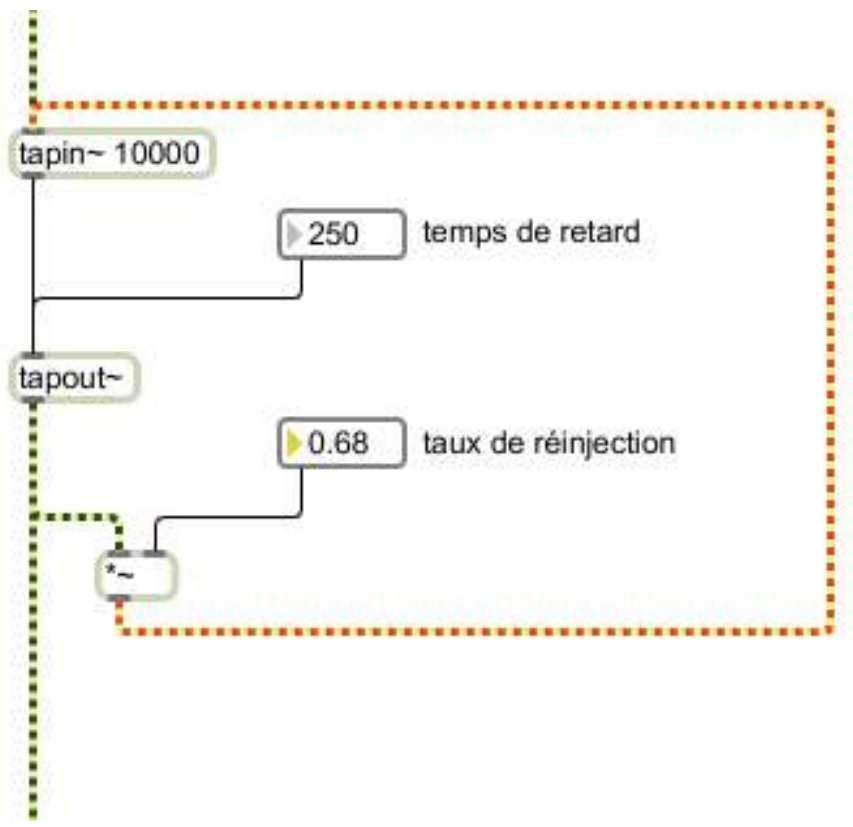

Il est possible d'effectuer quelques modifications à ce schéma général, afin d'introduire des variations timbrales dans le signal retardé. Une variation continue du temps de 
retard a pour effet une variation de la vitesse de lecture du signal retardé ; on peut ainsi, en introduisant une modulation non périodique du temps de retard, simuler l'effet de "pleurage " des échos à bande ${ }^{11}$. On peut également introduire un filtrage fréquentiel (passe-bande, par exemple) lors de la réinjection afin de simuler une dégradation du signal à chaque écho.

Figure 6a : modulation du temps de retard

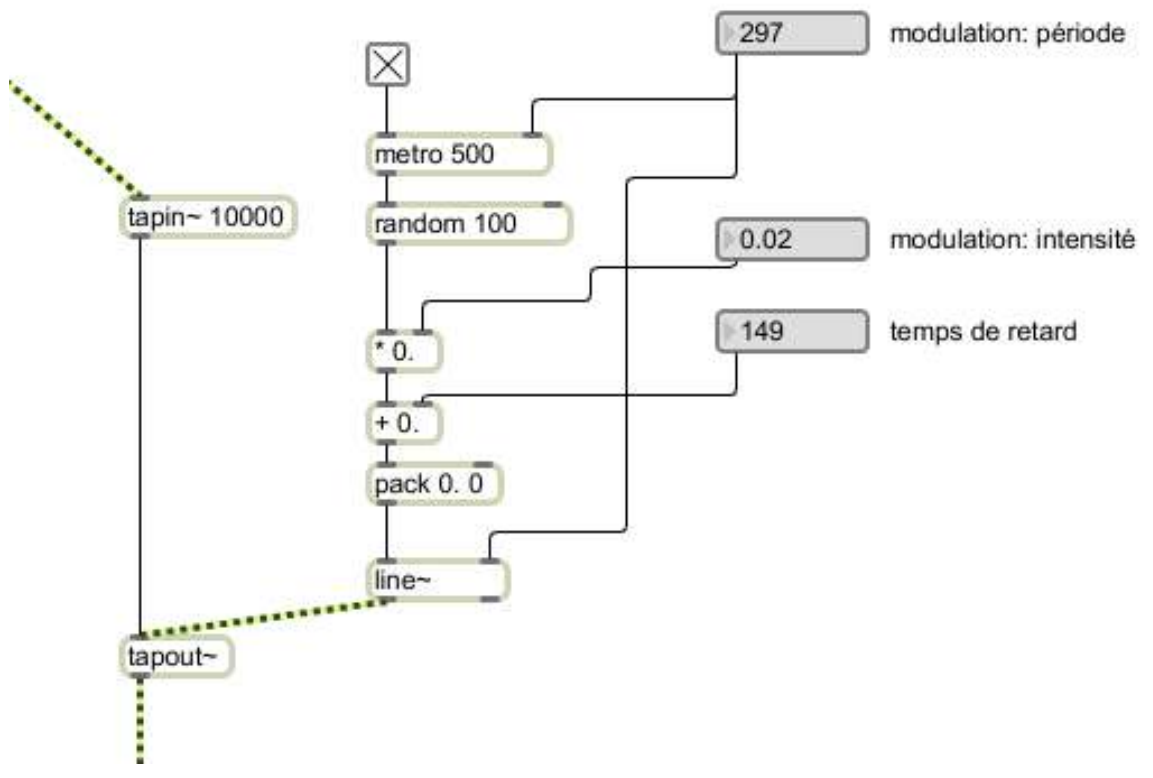

Figure $6 \mathrm{~b}$ : filtrage du signal dans la boucle de réinjection

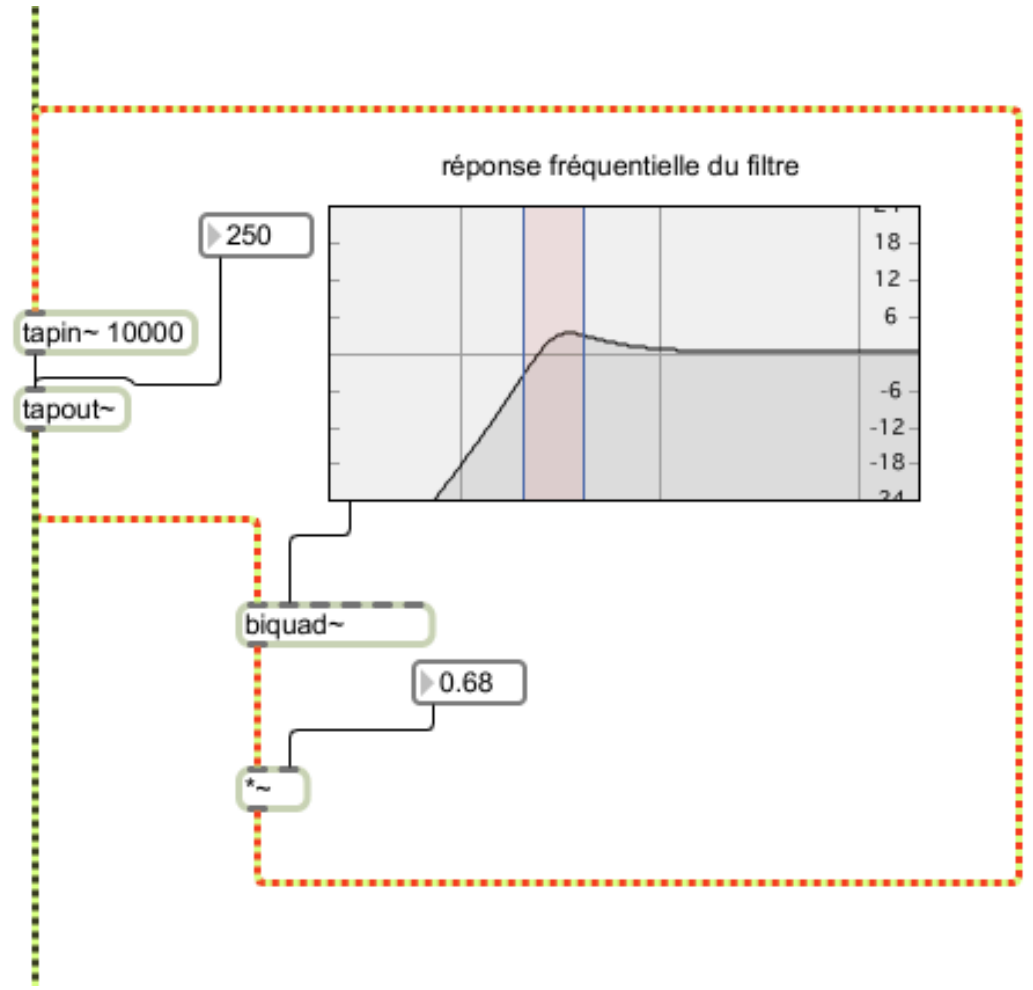



chorus et flanger en fonction du réglage de la balance entre son direct et son traité et du paramètre de réinjection :

- Le vibrato est obtenu pour une modulation périodique de quelques Hertz entre des valeurs très rapprochées de temps de retard, le son direct n'étant pas transmis en sortie.

- Le chorus est obtenu en rajoutant le son direct au schéma décrit pour le vibrato.

- Enfin, le flanger est obtenu en permettant la réinjection du signal. La fréquence de modulation peut alors descendre à quelques fractions de Hertz.

\section{*Envelope filter/Dynamic wah}

Certains traitements effectuent une analyse du signal entrant afin de faire varier certains paramètres de manière dynamique. C'est le cas avec les filtres fréquentiels pour l'Envelope Filter et la Dynamic wah. Il convient de distinguer les 2 cas suivants :

- L'Envelope Filter correspond à un filtre (passe-bas, en général) dont la fréquence de coupure varie selon une courbe prédéfinie (envelope, en anglais) et déclenchée lorsqu'une attaque est détectée dans le signal entrant. Nous proposons, pour ce cas-là, l'utilisation d'un éditeur graphique pour modifier la courbe de variation de fréquence de coupure.

\section{Figure 7 : édition de la courbe de variation de fréquence de coupure du filtre}
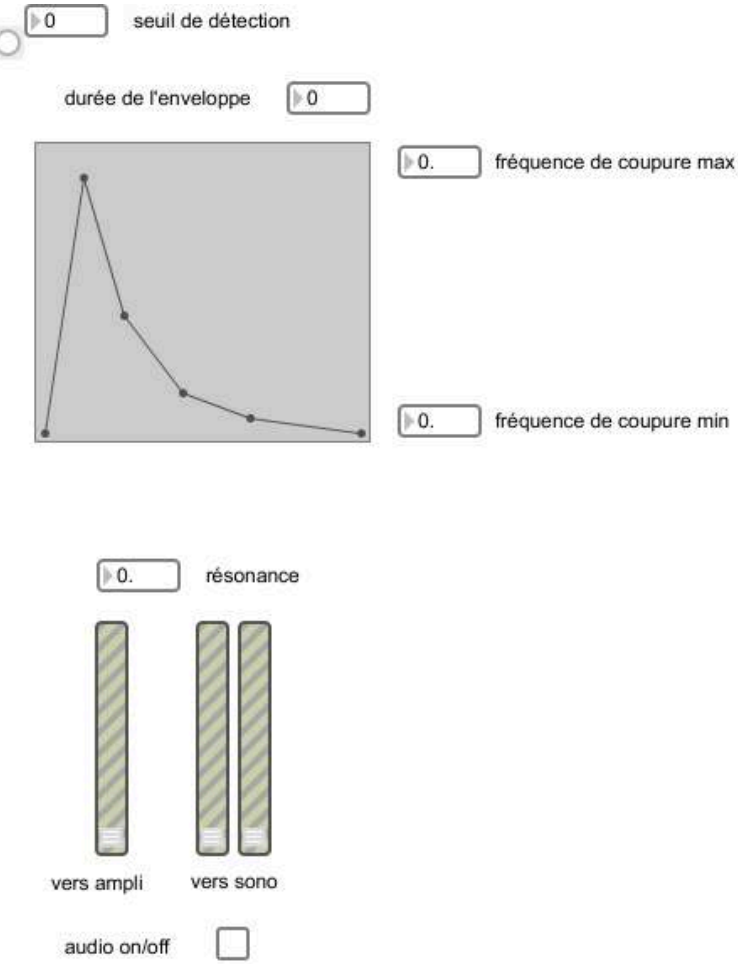

- Dynamic wah : ici, le niveau moyen du signal entrant sert à régler la fréquence de coupure du filtre. Nous avons développé un module autonome de suivi d'amplitude, intégrant une fonction de mapping permettant d'adapter l'échelle des données en sortie. 
Figure 8 : module de suivi d'amplitude, signal original (haut), résultat de l'analyse (bas)

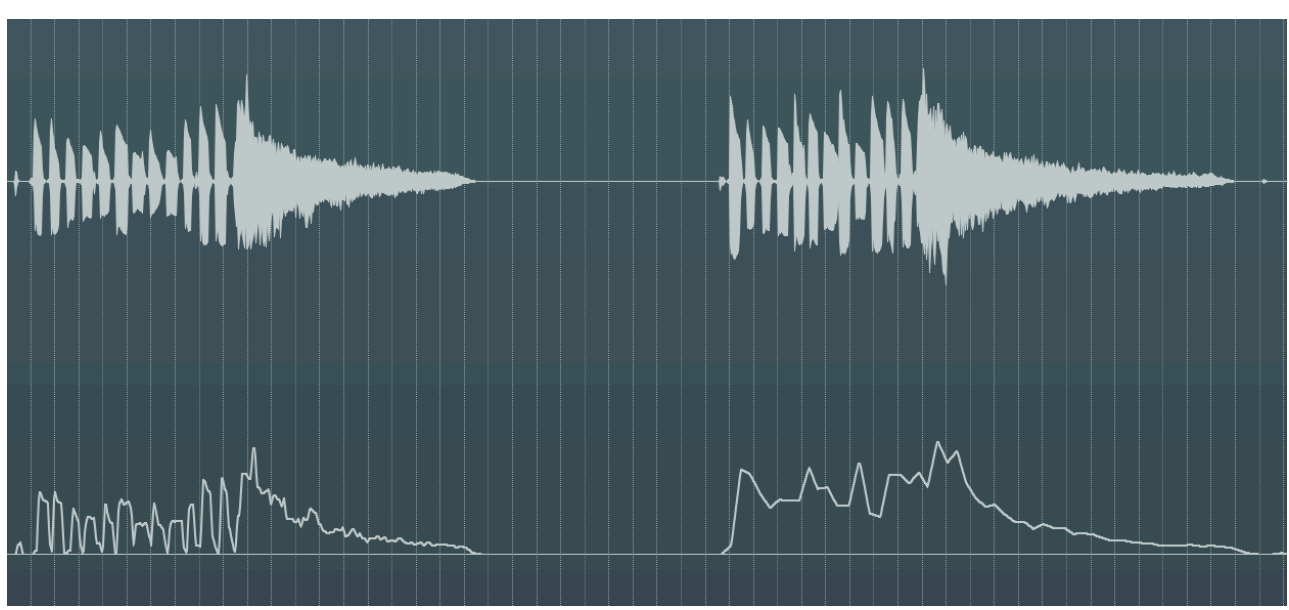

\section{*Dynamic tremolo}

Le module de suivi d'amplitude est ici utilisé pour piloter le paramètre de vitesse du Tremolo.

\section{*Tremolo, Delay, Chorus et Flanger multiphoniques}

Enfin, nous nous intéressons ici à la possibilité d'étendre les fonctionnalités de traitements vus précédemment pour une diffusion multicanal du signal audio.

Dans le cas du Delay, plusieurs lignes à retard transmettent le signal retardé à autant de canaux de sortie, une version incluant une matrice de réinjection est également proposée (le signal de chaque ligne à retard peut-être réinjecté dans les autres lignes à retard).

Dans le cas du Tremolo, du Chorus et du Flanger, on introduit un déphasage de $2 \pi / \mathrm{n}$ (où $\mathrm{n}$ est le nombre de canaux de sortie) entre les signaux de modulation de chaque canal, ce qui provoque une illusion de mouvement de source.

\section{Conclusion}

Dans cet article, nous avons présenté des modalités d'intégration de l'ordinateur comme outil de traitement du son pour la guitare électrique.

Après avoir présenté le système d'amplification de la guitare électrique, ainsi que la place que prennent les effets dans ce système, nous avons étudié les possibilités d'insertion de l'ordinateur dans ce système.

Enfin, nous avons présenté une série de traitements audionumériques développés dans l'environnement de programmation Max/MSP. 


\section{NOTES}

1. Si l'outil informatique peut également prendre une place dans le réseau sans intervenir sur le signal sonore (comme aide à la composition, ou simple magnétophone, par exemple), nous nous penchons plus particulièrement sur son implication dans la transformation du son de la guitare électrique.

2. http://www.cycling74.com

3. Certains préamplificateurs ne disposent que d'un réglage de gain, d'autres permettent, par le biais de filtres fréquentiels ou égaliseurs de modifier dans une large mesure le timbre de l'instrument.

4. Par exemple : le choix d'une technologie à tubes ou à transistors pour le préampli et l'ampli de puissance, le choix des composants eux-mêmes (différentes sonorités associées à différents tubes), choix du diamètre et de la marque du haut-parleur, réponse fréquentielle du hautparleur, nature et épaisseur du bois, topologie du baffle (open back, closed back...) sont autant de détails qui peuvent s'avérer primordiaux quant à leur influence sur la signature sonore de l'instrument.

5. Dans le cas du concert, l'amplificateur peut être sonorisé par un ou plusieurs microphones, le signal de ces microphones étant ensuite diffusé, après d'éventuels traitements, sur le dispositif de diffusion du concert. Ainsi, ce n'est pas le son direct de l'amplificateur qui est perçu.

6. Certains effets possèdent plusieurs sorties; c'est le cas de traitements stéréophoniques, par exemple, ou afin de disposer d'une sortie avec traitement et une sans traitement. On trouve également parfois plusieurs entrées, soit pour un traitement stéréophonique, soit pour des traitements permettant de moduler le signal à traiter avec un autre signal audio: entrée supplémentaire pour le circuit de sidechain d'un compresseur, par exemple.

7. On pourra également décider de se passer totalement de l'amplificateur de guitare et brancher directement la guitare dans l'interface audionumérique.

8. Certaines interfaces audionumériques disposent d'une entrée dite «instrument " ou « haute impédance » ou « hi-Z » permettant de connecter directement une guitare électrique.

9. Un réglage de la taille des blocs de traitement (ou buffers) via le pilote de l'interface audionumérique permettra d'agir sur la latence.

10. S'il est primordial, le contrôle des niveaux de signal transmis à la section de puissance n'est pas spécifique à l'utilisation de l'ordinateur.

11. L'effet de «pleurage » est dû à une variation de la vitesse de défilement de la bande.

\section{INDEX}

Mots-clés : amplification, traitement sonore, informatique musicale 
AUTEUR

BENOÎT COURRIBET

CICM - Université Paris 8, Maison des Sciences de l'Homme Paris Nord 